\title{
BigData Express: Toward Predictable, Schedulable, and High-Performance Data Transfer
}

\author{
BigData Express Research Team
}

September 18, 2019 


\section{Acknowledgments}

This work was supported by the U.S. DOE Office of Science ASCR network research program

U.S. DEPARTMENT OF

ENERGY
Office of

Science 


\section{BigData Express}

- BigData Express: a schedulable, predictable, and high-performance data transfer service

- A peer-to-peer, scalable, and extensible data transfer model

- A visually appealing, easy-to-use web portal

- A high-performance data transfer engine

- A time-constraint-based scheduler

- On-demand provisioning of end-to-end network paths with guaranteed QoS

- Robust and flexible error handling

- CILogon-based security 


\section{BigData Express Major Components}

- BigData Express Web Portal

- Access to BigData Express services

- BigData Express Scheduler

- Time-constraint-based scheduler

- Co-scheduling DTN, storage, \& network

- AmoebaNet

- Network as a service

- Rate control

- mdtmFTP

- High-performance data transfer engine

- http://mdtm.fnal.gov
- DTN Agent

- Manage and configure DTNs

- Collect \& report DTN configuration and status

Storage Agent

- Manage and configure storage systems

- 1/O estimation

- Data Transfer Launching Agent

- Launch data transfer jobs

- Support different data transfer protocols 


\section{BigData Express -- Distributed}

\section{Software Stack @ a BigData Express Site}

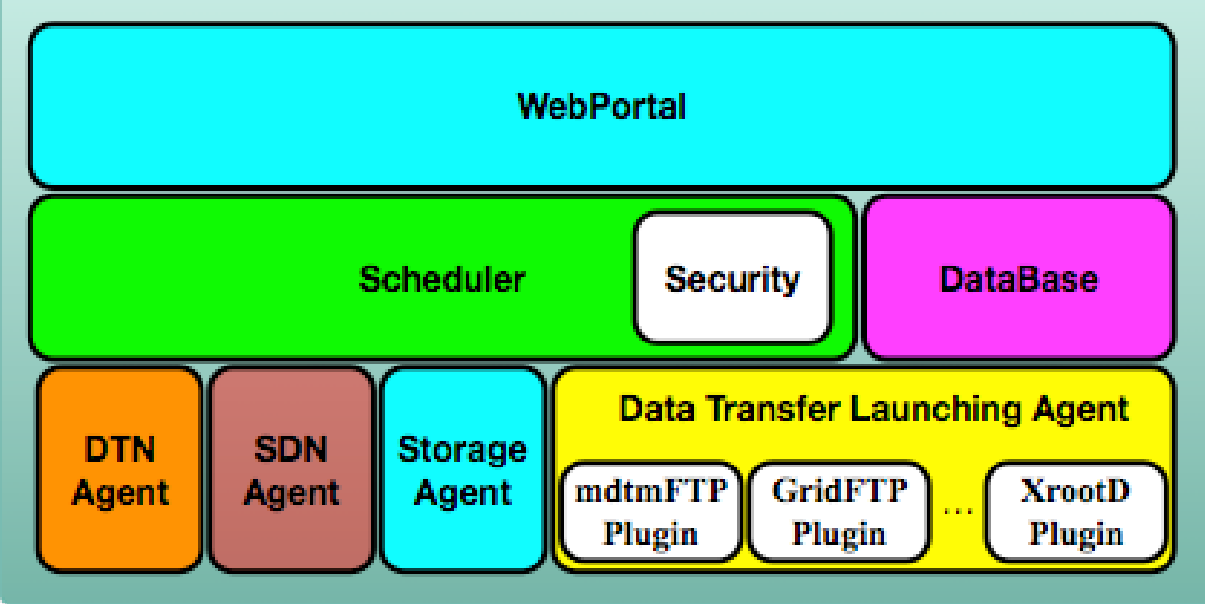

A Peer-to-Peer model

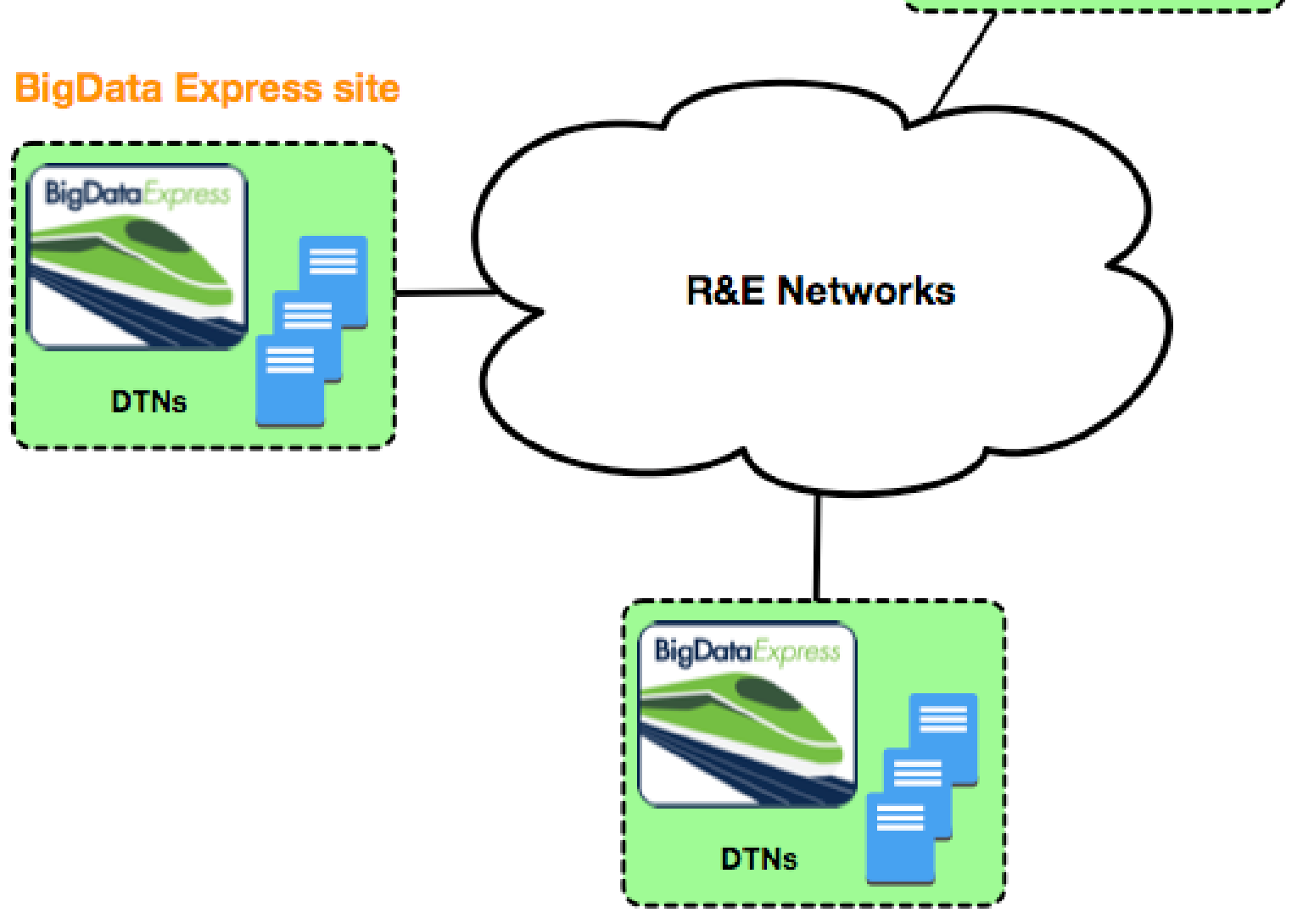

BigData Express site 


\section{BigData Express -- Flexible}

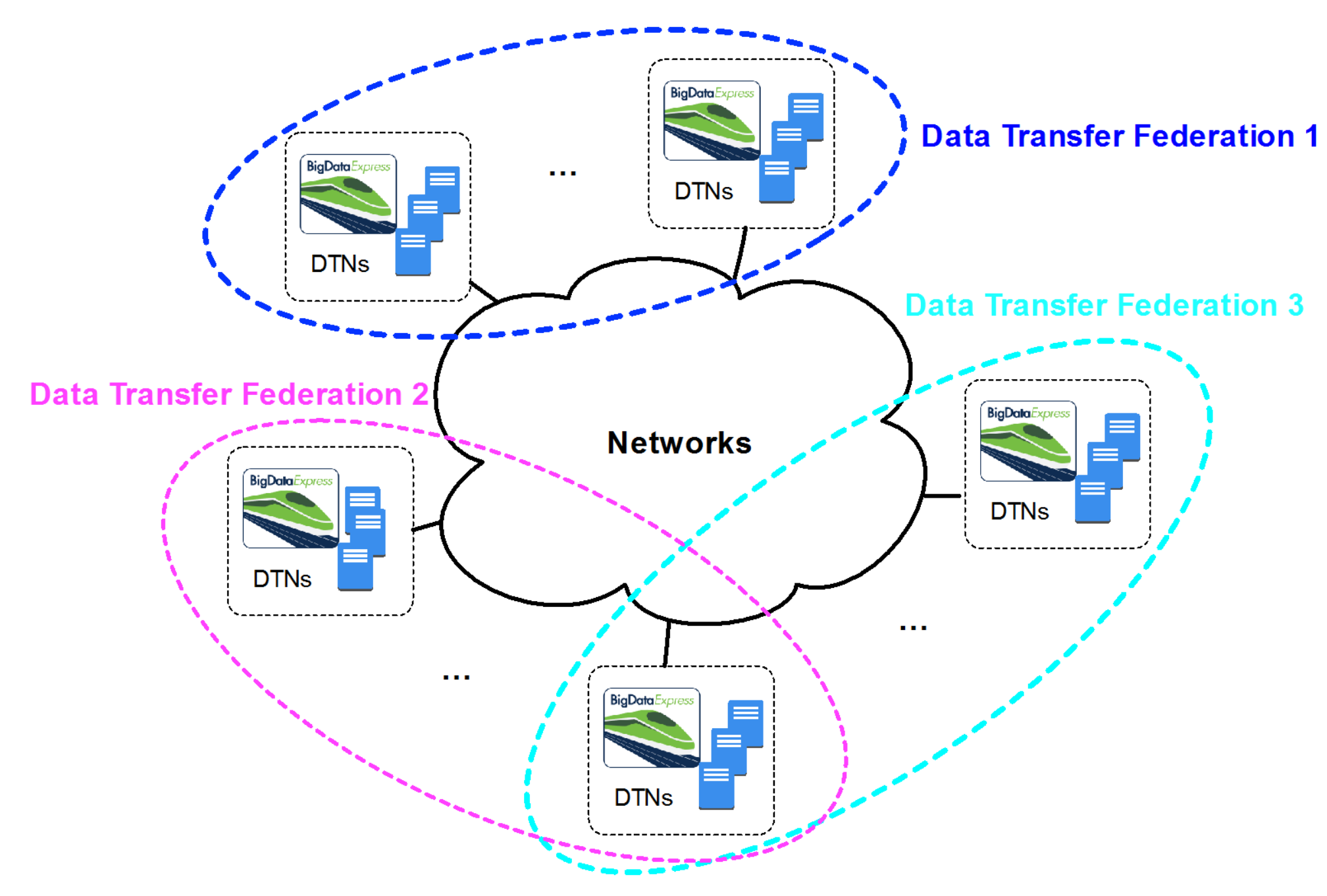

- Flexible to set up data transfer federations

- Providing inherent support for incremental deployment 


\section{BigData Express -- Scalable}

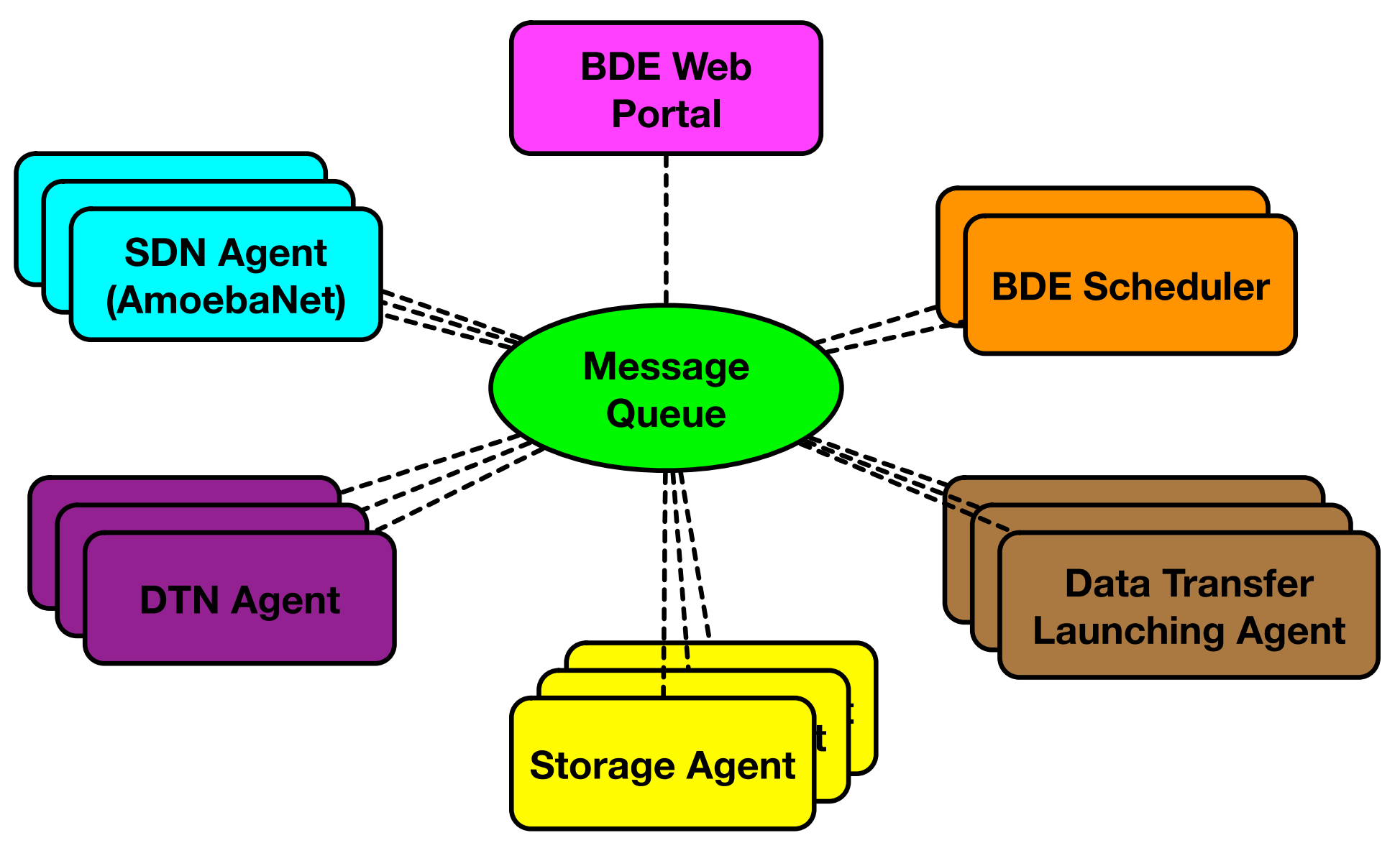

- BigData Express scheduler manages site resources through agents

- Use MQTT as message bus 


\section{BigData Express -- Extensible}

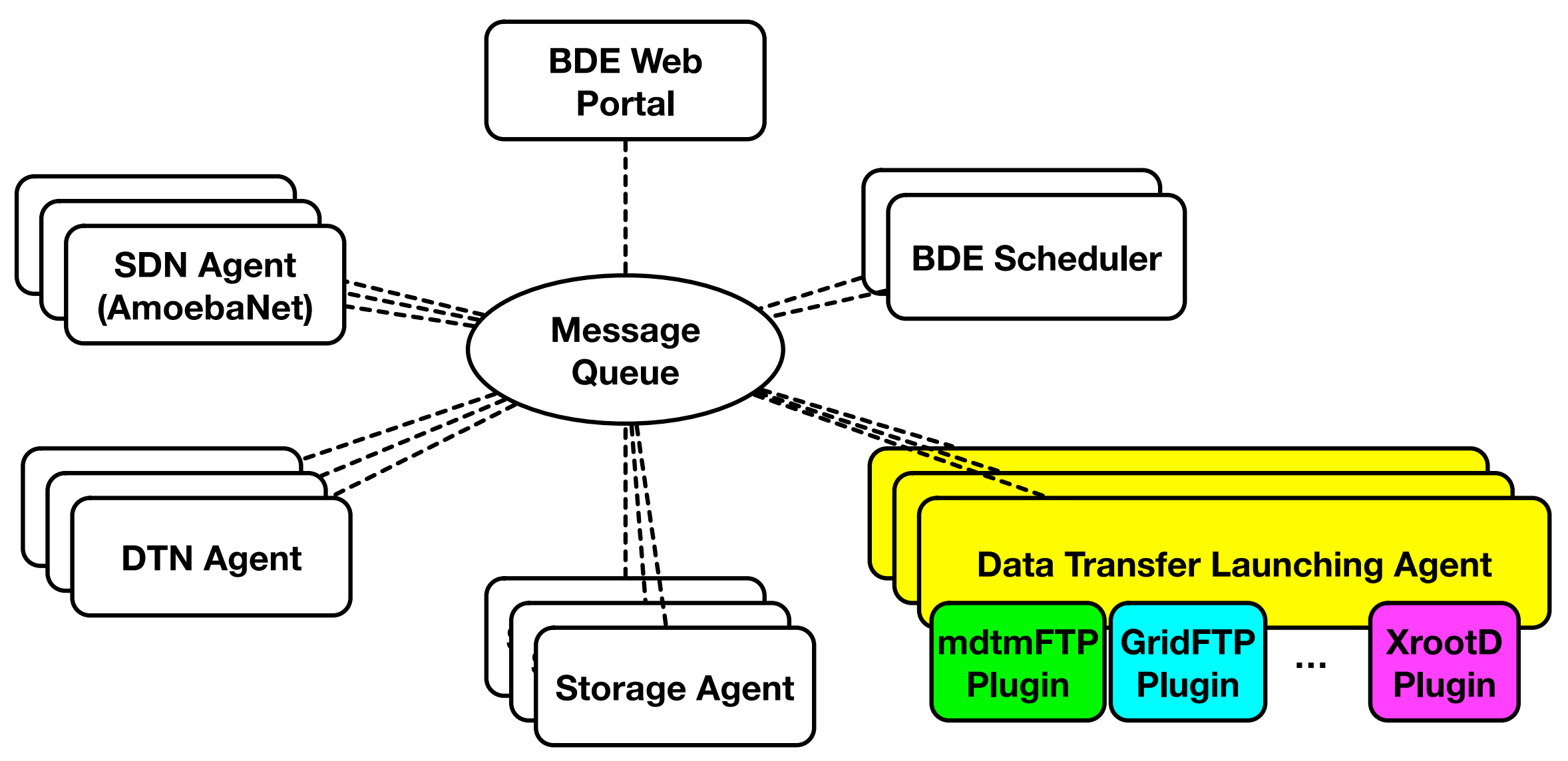

- Extensible Plugin framework to support various data transfer protocols - mdtmFTP, GridFTP, XrootD, ... 


\section{BigData Express -- End-to-End Data Transfer Model}

Site A - Smart E2E

Data Transfer Orchestrator

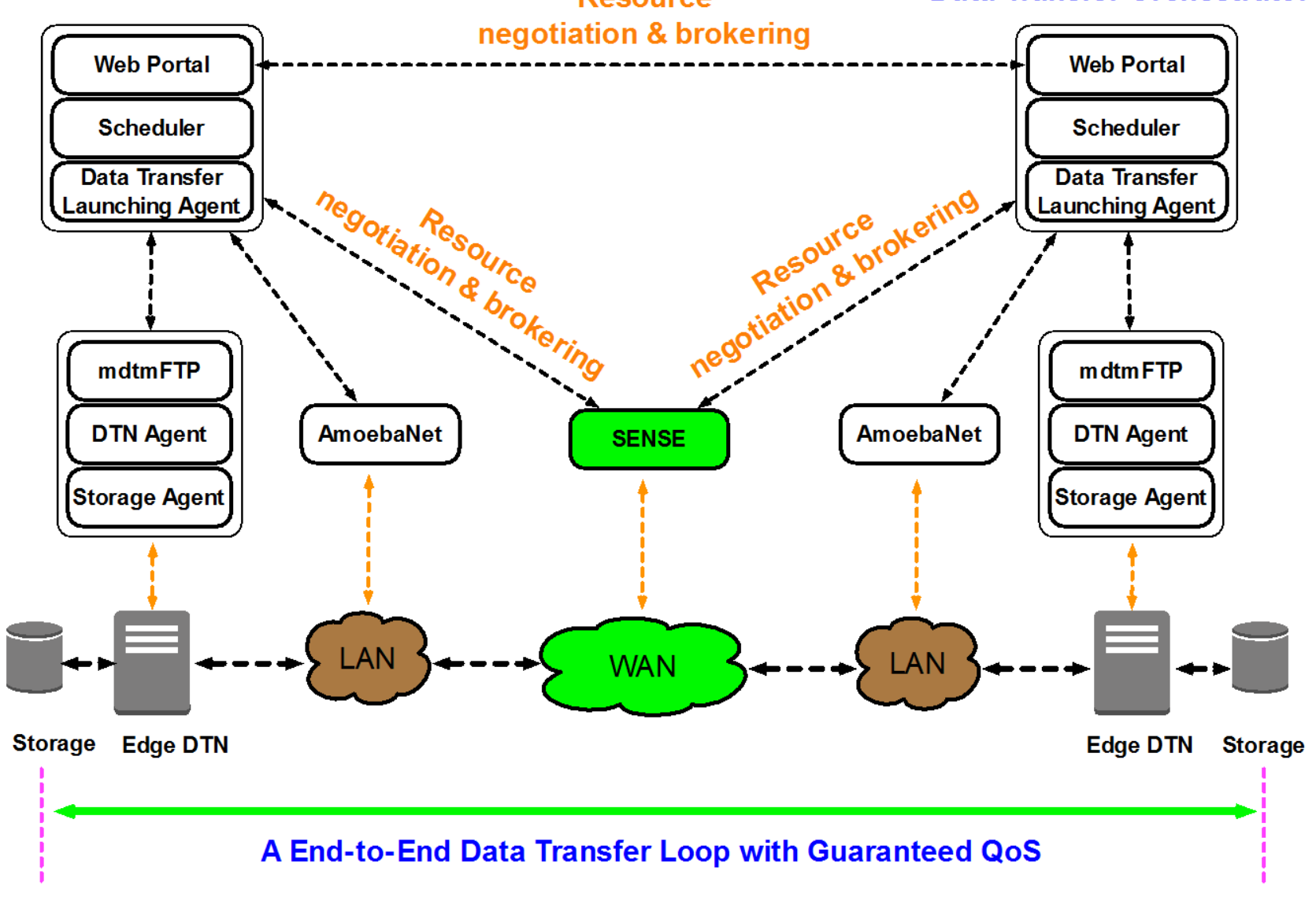

- Application-aware network service $\circ$ On-demand programming

- Fast-provisioning of end-to-end network paths with guaranteed QoS

- Distributed resource negotiation \& brokering 


\section{mdtmFTP \\ A high-performance data transfer tool}

- Pipelined I/O-centric design to streamline data transfer

- Multicore-aware data transfer middleware (MDTM) optimizes use of underlying multicore system

- Extremely efficient in transferring of Lots Of Small Files

- Various optimization mechanisms

- Zero copy

- Asynchronous I/O

- Batch processing

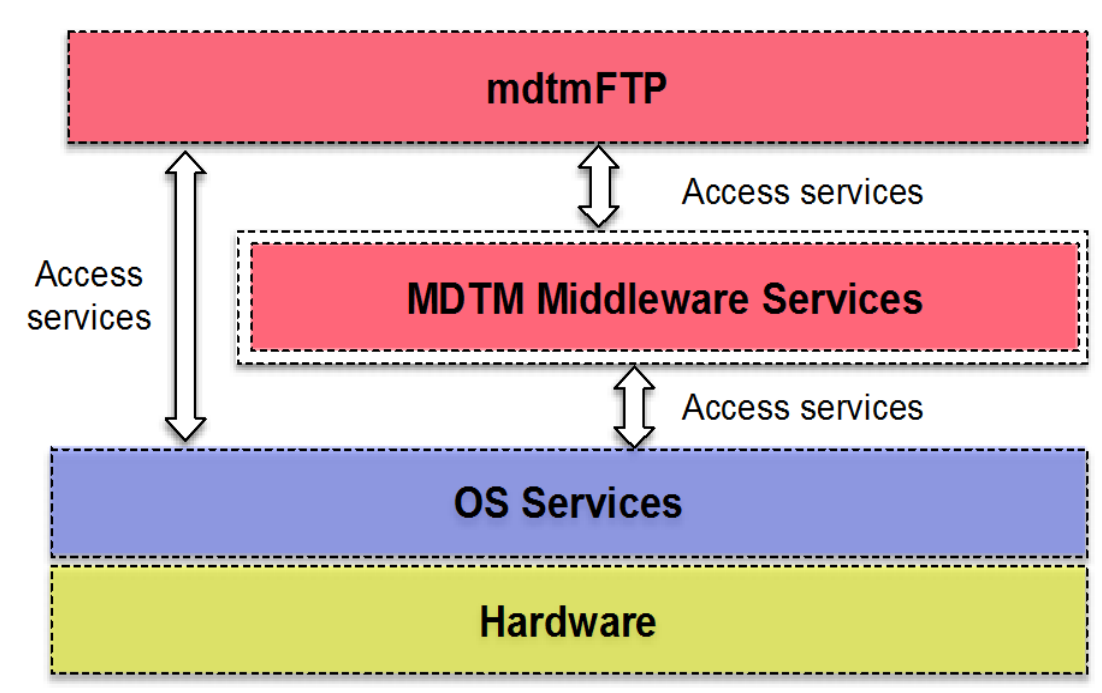




\section{BigData Express SC18 DEMO}

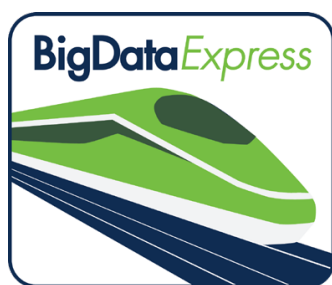

\section{苚 Fermilab}

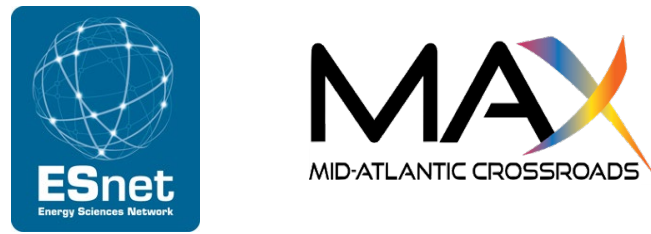

\section{OAK RIDGE \\ National Laboratory}
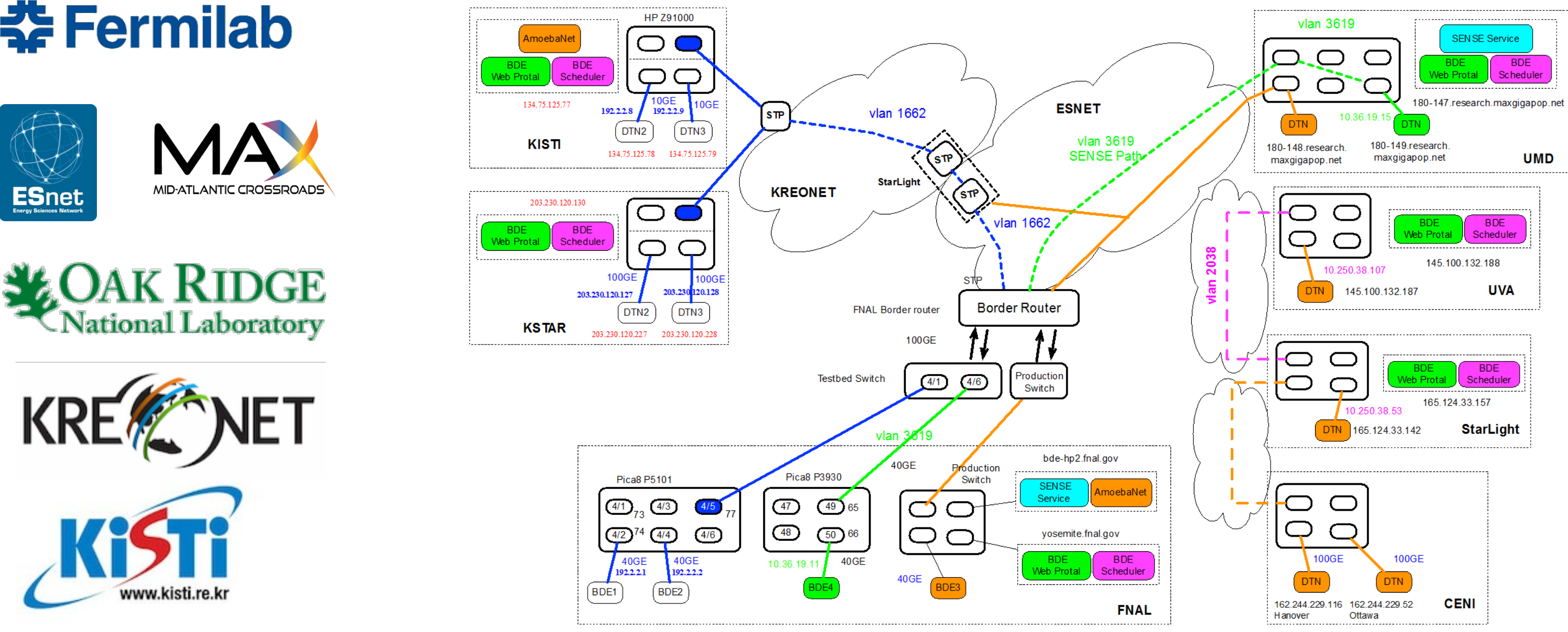


\section{BigData Express - Deployment}

- Asia

- KISTI, South Korea

- KSTAR

- Europe

- University of Amsterdam, Netherlands

- North America

- Fermilab

- StarLight, Northwestern University

- UMD/MAX, University of Maryland, College Park

- Ciena (Canada)

\section{KiSTI KBTAR}

- US East

- CA East

SURF NET

葓 Fermilab

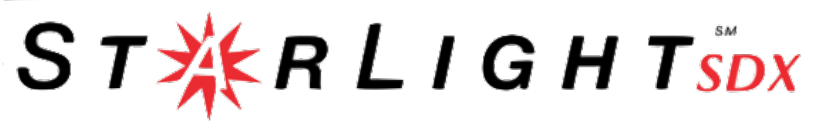

$M A$

ciena

Experience. Outcomes.

- Australia \& Pacific areas

- National Computational Infrastructure (NCl)

$\mathrm{NCl}$ 


\section{BigData Express Release}

- License

- Apache 2.0

- Docker release

-http://xxxxx

- Source code package release

-Available upon request 


\section{BigData Express Documents}

- BigData Express Admin Manual

- https://bigdataexpress.fnal.gov/bde install manual/index.html

- BigData Express Design and Architecture

-https://bigdataexpress.fnal.gov/bde design manual/index.html

- BigData Express Web Portal User Manual

-https://bigdataexpress.fnal.gov/bde portal manual/index.html 


\section{BigData Express Roadmap}

- REST APIs support for scientific workflows

- Support IPv6

- Support Kubernetes

-Automating docker-based deployment 


\section{More information about BigData Express}

\section{http://bigdataexpress.fnal.gov}

\section{Contact: wenji@fnal.gov}

This document was prepared by BigData Express using the resources of the Fermi National Accelerator Laboratory (Fermilab), a U.S. Department of Energy, Office of Science, HEP User Facility. Fermilab is managed by Fermi Research Alliance, LLC (FRA), acting under Contract No. DE-AC02-07CH11359. 